\title{
Moving Digital Disease Detection From Research to Action: Findings from a Survey of ISDS Membership
}

\author{
Jennifer Olsen* \\ University of North Carolina-Chapel Hill, Chapel Hill, NC, USA
}

\section{Objective}

Share the results of a survey on knowledge and attitudes regarding digital disease detection amongst ISDS members and share the resulting framework.

\section{Introduction}

Information is imperative to detecting and intervening in a disease outbreak, but the enormous amount of information that public health leaders must sift through can become overwhelming and obstructive. In the disease outbreak environment, it is imperative to understand which sources of information add value and should be used for decision making in this limited timeframe. Recent research has found that social media sources and news media sources may provide indicators of disease outbreaks prior to traditional reporting sources (i.e. surveillance systems) (1). WHO uses informal information sources for about $65 \%$ of their outbreak investigations and relies on informal sources for daily surveillance activities (2).

\section{Methods}

A computerized, self-administered questionnaire was developed using UNC's Qualtrics Research Suite to gather responses to an anonymous survey assessing likelihood of public health decision makers using various types of infodemiology data. Most questions had quantifiable responses using Likert scales only, except for the last two questions that were free form text. A grid format was used as often as feasible to eliminate redundancy, make the survey appear shorter, and require less effort from the participant - each of which may potentially contribute to improved data quality (3). The survey was distributed to the membership email list of ISDS. For the analysis of the survey responses, the data were made available as a dataset with categorical values assigned to each response. A descriptive analysis of findings was done to look at each question individually. For the two open-ended survey questions, each response was reviewed and if multiple concepts were in a single response those concepts were treated individually. Upon reviewing all responses, themes were identified and each response was categorized under one of these themes. Each set of responses within a theme was reviewed to identify key characteristics of the response, and these are the points that are included in the results.

\section{Results}

Amongst survey respondents, $80 \%$ indicated they were always looking for new types of data and information sources to inform public health response actions. There was more variation in the question focused on the possibility of getting too much information during an event. Responses were mixed from 6\% strongly agreeing, to $38 \%$ agreeing, $25 \%$ disagreeing, $15 \%$ strongly disagreeing, and $15 \%$ unsure. For most survey respondents, digital disease content had the most value in the first stage of situational awareness for identifying early indications of disease outbreaks. News media and internet searches were moderately to highly valuable for $70 \%$ of respondents, while social media was moderately to highly valuable to $60 \%$ of respondents. For both strengthening comprehension of an outbreak and informing future predictions, beliefs were split regarding the level of potential value (if any) that exists. When looking at digital disease content to identify early signals of disease outbreaks, all three potential sources were seen as moderately valuable by about $40 \%$ of survey participants. The distinction is between social media, seen as highly valuable by $18 \%$ and both news and internet search seen as highly valuable by $28 \%$. When assessing the value of social media for strengthening comprehension of an emerging outbreak, social media was most often seen as minimally valuable by participants.

\section{Conclusions}

To enable informed use of limited resources, the author used these survey results to develop a framework for incorporating digital disease detection into public health situational awareness practice.

\section{Keywords}

digital disease detection; situational awareness; biosurveillance

\section{Acknowledgments}

Courtney Corley, $\mathrm{PhD}$

Kristen Lich, PhD

Thomas Ricketts III, PhD

Gary Rozier, MD

Craig Vanderwagen, MD

\section{References}

1. Nelson NP, Brownstein JS, Hartley DM. Event-based biosurveillance of respiratory disease in mexico, 2007-2009: Connection to the 2009 influenza A(H1N1) pandemic? Euro Surveill. 2010 Jul 29;15(30):19626.

2. Heymann DL, Rodier GR, WHO Operational Support Team to the Global Outbreak Alert and Response Network. Hot spots in a wired world: WHO surveillance of emerging and re-emerging infectious diseases. Lancet Infect Dis. 2001 Dec;1(5):345-53.

3. de Leeuw ED, Hox JJ, Dillman DA, European Association of Methodology. International handbook of survey methodology. New York: L. Erlbaum Associates; 2008.

\footnotetext{
*Jennifer Olsen

E-mail: jenolsen.drph@gmail.com
} 